\title{
Pseudo tumour cerebri in systemic lupus erythematosus
}

\author{
Guwani Liyanage $^{1}$, H M D Herath ${ }^{2}$ \\ Sri Lanka Journal of Child Health, 2008; 37: 59-60
}

(Key words: Pseudo tumour cerebri, systemic lupus erythematosus)

\section{Introduction}

Systemic lupus erythematosus (SLE) is a multisystem autoimmune disorder characterised by a broad range of manifestations and the finding of antibodies in the blood directed against one or more components of cell nuclei ${ }^{1}$. The American Rheumatic Association (ARA) recommends 4 of the following 11 revised criteria for the diagnosis of SLE: malar rash, discoid rash, photosensitivity, oral ulcers, arthritis, serositis, renal disorder, neurologic disorder, haematologic disorder, immunologic disorder on serologic testing, and antinuclear antibodies ${ }^{2}$.

Neuropsychiatric manifestations can occur in up to two-thirds of patients with SLE. Pseudotumour cerebri (PC) is an exceedingly rare manifestation of neuropsychiatric SLE and one of the causes of headache in SLE patients. It is characterised by an elevated intracranial pressure, papilloedema with occasional abducent nerve paresis, absence of a space-occupying lesion or ventricular enlargement, and normal cerebrospinal fluid chemistry and cell count. If this condition is not treated promptly it can even lead to blindness. This has been reported in a few adult patients.

\section{Case report}

A 12-year-old girl from Ambalangoda was admitted to the professorial paediatric unit in Colombo South Teaching Hospital in September 2006 with intermittent fever and headache of 2 week duration. She also had episodic small joint pain, which started three weeks prior to onset of fever. She was seen by a general practitioner twice and treated with non steroidal anti inflammatory drugs (NSAIDs) for a presumed diagnosis of reactive arthritis. She had an unremarkable family history and past history.

${ }^{1}$ Senior Lecturer, Department of Paediarics, Faculty of Medical Sciences, University of Sri Jayawardenapura, ${ }^{2}$ Registrar in Paediatrics, Colombo South Teaching Hospital.

(Received on 18 April, 2007. Accepted on 20 May 2007 )
On examination, she was mildly pale, febrile but well looking. She had a faint butterfly shaped rash on the face and a few oral ulcers. Blood pressure was within the normal range. Examination of fundi revealed bilateral gross papilloedema (Figure 1) with minimally affected vision. Rest of the neurological examination was normal.

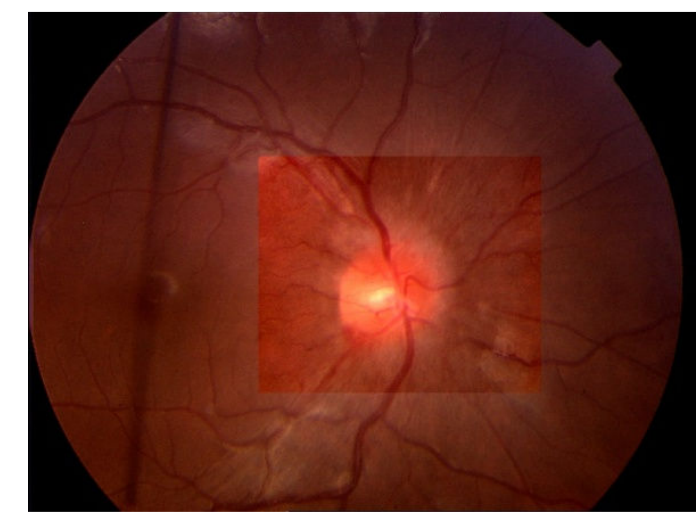

Figure 1 Fundal photograph showing gross papilloedema

Investigations revealed low platelets $\left(98 \times 10^{9} / \mathrm{L}\right)$, low haemoglobin $(7.9 \mathrm{~g} / \mathrm{dl})$, high ESR (98mm first hour) and a normal CRP. Blood picture showed normocytic normochromic red cells with thrombocytopenia and leucopenia. In addition, she had microalbuminuria with normal renal functions. Her antinuclear antibodies and double stranded DNA were positive. MRI scan of brain revealed mild cerebral atrophy without evidence of hydrocephalus or space occupying lesion. According to the ARA criteria a diagnosis of SLE was made and the patient started on high dose prednisolone.

A few days later she developed a generalised vasculitic rash and in addition renal functions started deteriorating with hypertension, gross proteinuria and generalised oedema. Creatinine clearance was $64 \mathrm{ml} / \mathrm{min} / 1.73 \mathrm{~m}^{3}$ and renal biopsy showed membranoproliferative glomerulonephritis. She was started on mycophenolate mofetil in addition to prednisolone. On follow-up she showed improvement in clinical and biochemical parameters and had complete recovery of pseudotumour cerebri. 


\section{Discussion}

Headache is a common symptom in SLE patients. Different types of headache may occur in SLE due to various underlying conditions. Pseudotumour cerebri (PC) is a rare manifestation of neuropsychiatric SLE and one of the causes of headache in SLE patients. Early detection and treatment is important since it can lead to permanent visual impairment.

Diagnosis of PC requires evidence of increased intracranial pressure after excluding a spaceoccupying lesion or hydrocephalus. Our patient had headache, vomiting and bilateral papilloedema suggestive of increased intracranial pressure. Magnetic resonant scanning of brain excluded a space occupying lesion or hydrocephalus. Therefore a diagnosis of PC was made.

Certain drugs (vitamin A, tetracycline, nalidixic acid, nitrofurantoin, sulpha derivatives, lithium, phenytoin and indomethacin, steroids, oral contraceptives) endocrinological abnormalities (hypo- and hyperthyroidism, hypoparathyroidism, adrenal insufficiency, Cushing's syndrome) and hypertension are known causes of PC.

Our patient did not have endocrinopathies; she was not on antibiotics or vitamins, which are known to induce PC. She did not have hypertension on initial presentation. Corticosteroid therapy could not be implicated because this patient was not receiving prednisolone at the time of diagnosis of PC. Therefore pseudo tumour cerebri in this patient was considered as a manifestation of neuropsychiatric SLE.

The pathophysiologic mechanism of PC in SLE remains unknown. It is not clear whether the overproduction of CSF or relative absorptive failure of CSF is associated with the pathogenesis of $\mathrm{PC}$ in $\mathrm{SLE}^{3}$. On the other hand, $\mathrm{PC}$ in SLE has been attributed to cerebral venous thrombosis in some cases. The most critical sequel of $\mathrm{PC}$ is the permanent visual impairment.
Corticosteroid is currently the mainstay of the treatment of $\mathrm{PC}$ in patients with $\mathrm{SLE}^{4}$. In addition, azathioprine, cyclophosphamide and other immunosuppressive agents can be used in the treatment of such patients.

We described a girl with SLE who presented with severe headache. Headache is a common symptom encountered in patients with neuropsychiatric SLE. Pseudo tumour cerebri needs to be considered in the differential diagnosis for headache in these patients.

\section{Acknowledgements}

We thank Dr. Champa Banagala, Consultant Eye Surgeon, Eye Hospital Colombo for the assistance given in doing the fundal photograph.

\section{References}

1. Mills JA. Systemic lupus erythematosus. New England Journal of Medicine 1994; 330: 18719 .

2. Tan EM, Cohen AS, Fries JF, et al. The 1982 revised criteria for the classification of systemic lupus erythematosus. Arthritis and Rheumatism 1982; 25: 1271-7.

3. Li EK. Pseudotumour cerebri in systemic lupus erythematosus. Journal of Rheumatology 1989; 16: 113-6.

4. L, Vinker S, Amital H, Amir T, Bar-Dayan Y, Levi Y, SchoenfeldY. Pseudotumour cerebri in systemic lupus erythematosus. Seminars in Arthritis and Rheumatism 1995; 25: 103-8. 